\title{
Cynan a rocfeirdd y Clarence Hotel, 1924
}

Wiliams, Gerwyn

\section{Llên Cymru}

DOI:

$10.16922 / \mathrm{lc} .42 .8$

Cyhoeddwyd: 01/10/2019

Fersiwn wedi'i hadolygu gan gymheiriaid

Cyswllt i'r cyhoeddiad / Link to publication

Dyfyniad o'r fersiwn a gyhoeddwyd / Citation for published version (APA):

Wiliams, G. (2019). Cynan a rocfeirdd y Clarence Hotel, 1924. Llên Cymru, 42, 141-162.

https://doi.org/10.16922/lc.42.8

\section{Hawliau Cyffredinol / General rights}

Copyright and moral rights for the publications made accessible in the public portal are retained by the authors and/or other copyright owners and it is a condition of accessing publications that users recognise and abide by the legal requirements associated with these rights.

- Users may download and print one copy of any publication from the public portal for the purpose of private study or research.

- You may not further distribute the material or use it for any profit-making activity or commercial gain

- You may freely distribute the URL identifying the publication in the public portal ?

Take down policy

If you believe that this document breaches copyright please contact us providing details, and we will remove access to the work immediately and investigate your claim. 


\section{CYNAN A ROCFEIRDD Y CLARENCE HOTEL, 1924*}

Fel bardd, cafodd Cynan ryfel da a gwnaeth ei enw yn ei sgil. O'r beirdd eraill a ymatebodd i'r Rhyfel Byd Cyntaf, roeddent i gyd yn brifeirdd sefydledig cyn hynny: T. Gwynn Jones, R. Williams Parry, W. J. Gruffydd. O ran ei ddwyn i amlygrwydd cenedlaethol, Hedd Wyn yw'r un a ddaw agosaf ato, ond fyth ers Eisteddfod Aberystwyth yn 1916 pan ddaeth yn ail am y gadair, roedd ei enw yn y ffrâm, fel petai, ac er i'w gwymp yng Nghefn Pilkem ddiwedd Gorffennaf 1917 a'i fuddugoliaeth gydag awdl 'Yr Arwr' yn Eisteddfod Birkenhead ym Medi'r un flwyddyn gadarnhau ei enwogrwydd fel eicon cenedlaethol, diweddodd yr un pryd ei yrfa fel bardd. Cynan yw'r unig fardd Cymraeg o bwys y cafodd ei yrfa genedlaethol ei lansio gan y rhyfel a cheir awgrym o'r ffordd y daliodd ei gerddi sylw yng ngeiriau E. Morgan Humphreys yn ystod y rhyfel. Dwy gerdd ryfel gyntaf Cynan i'w cyhoeddi yn y Welsh Outlook oedd 'Ym Min y Môr' ac 'Anfon y Nico' a phan gynhwyswyd y ddwy ym mlodeugerdd Annie Ffoulkes, Telyn y Dydd, yn 1918, mewn llythyr personol at T. Gwynn Jones, canmolodd y newyddiadurwr yn benodol gerddi ei gyfaill, rhai R. Williams Parry a rhai 'Cynan, pwy bynnag ydyw'. Hynny yw, denodd glod yn ddiragfarn ar sail ei gynnyrch yn hytrach na'i enw. ${ }^{1}$

I'n hatgoffa o ddatblygiad sydyn yr yrfa honno, yn arena'r Eisteddfod Genedlaethol yn y Barri yn 1920, daeth yn fuddugol 'am gyfrol lenyddol (Belles Lettres) mewn barddoniaeth neu ryddiaith'; ${ }^{2}$ wrth galon y casgliad hwnnw o gerddi roedd y saith a gyhoeddwyd ar dudalennau'r Welsh Outlook rhwng Hydref 1917 a Medi 1919. Ac yntau wedi cael blas ar lwyddiant, troes ei olygon nesaf at goron y Genedlaethol, ac er cwyno'r beirniaid am ei rhythmau agoriadol clogyrnaidd ac ambell olygfa styrbiol, go brin fod yr un bryddest eisteddfodol wedi profi'r un poblogrwydd â phryddest 'Mab y Bwthyn' yn Eisteddfod Caernarfon yn 1921. Ond nid cerdd o ddiddordeb llenyddol mohoni'n unig: daliodd y bryddest, yn enwedig y rhannau realaidd ohoni, ysbryd yr oes fel yr awgrymir gan sylw un colofnydd papur newydd a fynnai y dylid '[c]odi cymdeithas diarfogiad yn y wlad, a galwer hi'n "Gymdeithas Mab y Bwthyn". ${ }^{3}$ Gorseddwyd Cynan yn lladmerydd ei genhedlaeth, un o oroeswyr ifainc dewr y rhyfel a ddaeth yn ôl i adrodd ei hanes a'i stori ei hun a chynnig gobaith a gyferbynnai â thrasiedi stori Hedd Wyn.

Wrth iddo dafoli ei yrfa yn 1987, yr hyn a welodd Bedwyr Lewis Jones oedd '[p]rydydd ifanc gwrthryfelgar - ffigur pwysig yn llenyddiaeth Cymru yn 1917-20 - a barchusodd yn llawer 
rhy gynnar fel bardd'. ${ }^{4}$ Ym mhapur wythnosol y North Wales Weekly News yn y 1920au, cyfeirir ato droeon a hynny braidd yn lletchwith fel 'Rev. A. E. Jones (“Cynan”)' sy'n awgrymu personoliaeth hollt neu hunaniaeth ddwbl. Dyna a awgrymir gan eiriau Bedwyr hefyd sef deuoliaeth rhwng y Parch. Albert Evans Jones cyhoeddus a Cynan y bardd. Oherwydd i ifainc radical y 1960au, roedd Cynan yn ymgorfforiad o barchusrwydd sefydliadol, nodwedd a grisialwyd gan ei frenhiniaeth dryloyw. Ychydig dros chwe mis cyn ei farw yn Ionawr 1970, fe'i gwelid yn ei elfen yn rhan o'r osgordd Orseddol yn seremoni arwisgo'r Tywysog Siarl yng Nghastell Caernarfon; lediodd emyn yn y seremoni rwysgfawr honno a'i wobrwyo am ei ran yn nhrefniadau'r achlysur drwy'i ddyrchafu'n farchog; a phan ddaeth yr Eisteddfod Genedlaethol i'r Fflint fis yn ddiweddarach a Syr Cynan erbyn hynny'n Llywydd y Llys, croesawodd y tywysog ifanc i lwyfan y brifwyl a daliwyd ei ddiléit tadol mewn lluniau ar y pryd. ${ }^{5}$

Ond at Cynan y bardd y cyfeiriodd Bedwyr ac er mai'r tair blynedd rhwng 1917 a 1920 a bwysleisir ganddo, fe ddaliwn i na fu'r parchuso yn ei hanes yn broses mor rhwydd â hynny ac na ellir tynnu llinell derfyn mor glir â dechrau'r 1920au. Y cerddi a heliwyd ynghyd yn Telyn y Nos yn 1921 a bwysleisir gan Bedwyr, ond mae lle i ystyried hefyd gerddi'r 1920au a gasglwyd ynghyd yn Caniadau Cynan yn 1927. Gan mor llachar yw'r darlun diwedd oes ohono fel cynheiliad y sefydliad a chefnogwr y frenhiniaeth, efallai ei fod wedi ein dallu a'n rhwystro rhag rhoi'r sylw dyladwy i'r anghonfensiynoldeb, yr annibyniaeth barn, y tynnu'n groes a throi'r drol, mewn gair, i'r amharchusrwydd sy'n nodweddu amryw o'i gerddi ar hyd y 1920au a'u bod yn haeddu ailddarlleniad. Yn sicr, mae fy ymchwil ddiweddar i’w fywyd a'i waith yn awgrymu fod ei awen yn fwy rhyddfrydig os nad radical yn y blynyddoedd ar ôl y rhyfel nag a feddylir fel arfer. Gellid bod wedi mabwysiadu'n deitl amgen i'r ddarlith hon 'Pa Bryd y Parchusodd Cynan?'. O ran ei ymarweddiad cyhoeddus, nid oedd amharchusrwydd yn talu'r biliau nac yn sicrhau to uwch ei ben, ac er bod ei farddoniaeth yn ffynhonnell incwm iddo ar hyd y blynyddoedd - a byddai'n gwarchod hawlfraint ei gerddi'n ofalus ac yn mynnu ei freindal - ni allai fyw ar ei enillion fel bardd yn unig. Roedd yn rhaid wrth swydd yn y byd a hynny fel gweinidog gyda'r Methodistiaid Calfinaidd, yr enwad y troes ato ar ôl cael ei ddadfyddino yn 1919 gan gefnu ar enwad ei fagwraeth, y Bedyddwyr. Yn ystod yr un mlynedd ar ddeg rhwng 1920 a 1931, bwriodd i ganol bywyd prysur ei ofalaeth yng nghapeli Jerusalem a'r Glyn ym Mhenmaenmawr a chwarae ei ran yn llawn yng nghymdeithas fyrlymus y dref honno yng nghysgod y graig ac yng ngolwg y môr sydd fel petai wedi ei ymestyn fel tamaid o lastig rhwng twnnel Pen-y-clip ar ochr Môn a 
Phenmaenbach ar ochr Llandudno. Drwy gydol ei weinidogaeth, tystia'r adroddiadau cyson yn y papur newydd lleol i'r modd y sianelodd lawer iawn o egni diwygiadol i mewn i'w ofalaeth, yn enwedig ei waith gyda'r ifainc. Er enghraifft, sefydlodd Urdd Pobl Ifanc Jerusalem a gyfarfyddai bob wythnos i drafod a dadlau myrdd o bynciau crefyddol a chymdeithasol. Mor fuan â Chwefror 1923 fe'i derbyniwyd yn aelod o gyfrinfa'r dref o'r Seiri Rhyddion, sefydliad y bu'n aelod ffyddlon ohono weddill ei oes. Pan holwyd ef yn Cyfarwyddiadur Awduron Cymraeg Cyfoes a gyhoeddwyd ym mlwyddyn ei farw i ba gymdeithasau y perthynai, ef oedd yr unig un o blith dros gant a enwodd y gymdeithas arbennig hon y dywedodd Stephen Knight amdani: 6 'Far from being revolutionary, there is no organization more reactionary, more Establishment-based, than British Freemasonary. Its members derive benefit from the Brotherhood only so long as the status quo is maintained.' 7 Mae hwnnw'n ddisgrifiad anodd ei gysoni gyda bardd a oedd wedi ei ragweld ei hun yn eironig mewn cerdd a gyhoeddwyd gyntaf yn Ionawr 1923 - fis yn unig cyn dod yn Saer Rhydd - yn profi 'Adlais o'r hen wrthryfel | A wybu f'enaid gynt', ${ }^{8}$ h.y. pan fyddai'n 'hen a pharchus', nid pan oedd yn saith ar hugain oed!

Bron o gychwyn cyntaf ei weinidogaeth, felly, amlygir tensiynau ac anghysonderau rhwng y Parch. A. E. Jones cyhoeddus a Cynan y bardd. Cyfeiriwn ato'n amhroblematig fel un a gynrychiolai ffenomen y bardd-bregethwr, ond po fwyaf y meddyliaf am y peth, ei weld yn ei chael hi'n fwyfwy anodd cysoni'r ddwy swyddogaeth a wnaf, a bod hynny'n un o'r ffactorau a arweiniodd yn 1931 at ei ymadawiad â'r weinidogaeth gyfundrefnol. Dehongliad angharedig fyddai awgrymu ei fod yn chwarae'r ffon ddwybig, ond gorsymleiddiad fyddai hynny a dylid gochel rhag y demtasiwn i ddehongli amgylchiadau'r 1920au gyda'r un ffon fesur â Chymru'r presennol. Yn ystod y 1920au roedd ynddo rwystredigaethau a dyheadau na châi hi'n hawdd eu mynegi ar goedd ac yntau'n gwisgo'r goler gron ac i'r dibenion hynny bu'n dda iddo wrth bersona'r bardd. Mae a wnelo'r rhwystredigaethau hynny'n rhannol ag enwadaeth ddiddychymyg a phiwritaniaeth grefyddol fel y'u mynegir yn 'Y Nasaread':

Darllenais am danat, fy Arglwydd,

Yn troi dwfr Cana yn win.

Ond clywaf Dy fod Di heddiw

Yn llwyrymwrthodwr blin. ${ }^{9}$ 
Yr hyn sy'n ddiddorol yma yw bod y feirniadaeth ar ddirwestaeth yn mynd yn groes i'r hyn y bu Cynan yn gyfarwydd ag ef yn ystod ei fagwraeth ym Mhwllheli, roedd yn dal yn bwnc cyfredol yn y 1920au a uniaethid gyda Rhyddfrydiaeth drwy gymeriad y llwyrymwrthodwr Lloyd George sef yr aelod seneddol lleol, ac roedd un o bennaf cyfeillion Cynan ym Mhenmaenmawr, Mrs Phillips-Williams, y cyflwynodd Caniadau Cynan iddi hi a'i gŵr, yn aelod amlwg o gangen leol Cymdeithas Ddirwest y Merched. Ond roedd Cynan y bardd eisoes wedi canu clodydd Mimi, 'angel yr estaminet', yn 1921 a mynnu ganddi'r 'Medoc gorau yn y tŷ', ${ }^{10}$ a gwyddai'r cyn-filwr dynol a chydymdeimladol o'r gorau nad oedd hi'n ddim syndod 'na ddaeth y bechgyn adref o Ffrainc fel "saint". ${ }^{11}$

Mynegir rhwystredigaeth Cynan hefyd yn 'Monastîr':

Cyfododd yntau'r Llywydd: “Mae'n bleser gen i'n awr Ofyn i'r diaconesau ein hannerch o'r Sêt Fawr."

Yr oedd eu gwisg yn barchus, a'u gwallt yn barchus-dynn, A pharchus eu cerddediad. ${ }^{12}$

Bron nad yw 'parchus' yn gondemniad gan Cynan, ac er gwisgo persona'r bardd, mae'r modd y gwneir cyff gwawd o barchusrwydd diaconesau y Cyfarfod Misol a'u cymharu'n anffafriol gydag ysbryd rhydd a synhwyrus Chlöe'r fugeiles - chwaer i Mimi ramantus - yn fentrus os nad rhyfygus i'r parchus barchedig weinidog. Felly hefyd y modd y rhydd fynegiant mor blaen ac ergydiol i gondemniad un o gleifion y Rhyfel Mawr ar Dduw yn 'Y Tannau Coll', y bryddest y methodd ag ennill ei ail goron genedlaethol â hi: 'Mae Duw yn fyddar. Mae Duw yn fud. | Mae Duw heb galon. Mae Duw yn ddall.' ${ }^{13}$ Ac roedd hyn, cofier, yn 1922 yn rhagddyddio'r gwacter ystyr a fynegai T. H. Parry-Williams yn 'Yr Esgyrn Hyn' (1923) a 'Celwydd' (1924). ${ }^{14}$ Un o'r rhesymau pam na lwyddodd Cynan yn Eisteddfod Rhydaman oedd fod y beirniaid yn gweld bai arno am rygnu ar thema'r rhyfel a hwythau'n ysu am gân newydd. Daeth Cynan o hyd i gân newydd yn 1923 a hynny yn hanes y Tad Damien, offeiriad Catholigaidd a aberthodd ei iechyd er lles gwahanglwyfion ynys Molocai. Ond wrth ddyrchafu arwriaeth Damien ym mhryddest fuddugol Eisteddfod yr Wyddgrug, nid oedd ei feirniadaeth ar enwadaeth yn bell o'r wyneb chwaith ac fe'i beirniadwyd ar y pryd. Mewn llythyr ym Medi 1923, bwriodd ei lach ar 'y rhai a ddilornai Damien am ei fod yn Babydd': 
Meddyliwch mewn difri am ddyn, a'i geilw'i hun yn 'Secretary of the Protestant Press Bureau' yn sgrifennu fel hyn ataf ar ol yr Eisteddfod: 'Dear Sir, Had you known anything worth knowing about Damien you would never have mentioned his name.' . . Minnau a atebais: 'With reference to your chivalrous comment on Father Damien's work, I commend for your perusal Robert Louis Stevenson's open letter to the Rev. Dr Hyde. Note particularly the closing sentence: "The man who did what Damien tried to do was my father, ... and the father of all who love goodness, and he was your father too, if only God had given you the grace to see it."' 15

Mae'n werth ein hatgoffa ein hunain fod sail egwyddorol ac ymarferol i'w ymadawiad â'r Bedyddwyr yn 1919, gwrthwynebiad i gymun caeth ac yntau wedi gweinyddu'r cymun i ddynion o sawl enwad Anghydffurfiol boed nhw wedi profi bedydd trochi ai peidio, ond hefyd y ffaith nad oedd olwg ar y pryd ar ofalaeth gyda'r Bedyddwyr heblaw ei fod yn gaethgymunwr. Tystiolaeth ei ddiweddar fab yng nghyfraith imi oedd ei fod yn y cyfnod hwn wedi ystyried mynd yn offeiriad eglwysig. Hynny yw, cynnig ateb amserol a wnaeth Methodistiaeth Galfinaidd yn hytrach na'i fod wedi troi ati oherwydd rhyw sêl fawr drosti. Agwedd ryddfrydig a phragmataidd a feddai tuag at enwadaeth, ac ymhen blynyddoedd, dehonglai ei safiad dros Damien fel arwydd cynnar o ecwmeniaeth.

Tra oedd y Parch. A. E. Jones yn ei ymwneud bob dydd ym Mhenmaenmawr yn cymysgu â'r hen a'r ifanc - roedd yn ffrindiau gyda'r prif ŵr busnes lleol, y Cyrnol C. H. Darbishire oedrannus, a oedd yn berchen ar y chwarel ithfaen leol ${ }^{16}$ - mae cerddi Cynan ar y pryd yn rhoi mynegiant hefyd i'w ieuengrwydd a'r tyndra rhwng yr ifanc a'r hen. Yn 'Aberdaron' a gyhoeddwyd gyntaf yn y Western Mail rhoes fynegiant i'w rwystredigaeth ar ôl cael ei gorddi gan sylwadau Meuryn, golygydd Yr Herald Cymraeg a Papur Pawb. Cofir mai Meuryn oedd cyd-brifardd Cynan yng Nghaernarfon yn 1921 pan enillodd y gadair gydag awdl 'Min y Môr', ond serch hynny, nid ymddengys bod fawr o frawdoliaeth farddol rhwng y ddau:

Roedd golygydd rhyw bapur Cymraeg, gŵr hen a pharchus iawn, wedi bod yn ymosod, yn annheg iawn i'm meddwl i, ar gerddi y beirdd ifainc. Roedd fy hen gyfaill Prosser Rhys a minnau'n ifanc iawn 'ramser hynny ... Ac yn fy nigofaint a minnau'n darllen y peth yn y papur newydd ar fy ngwyliau yn Aberdaron yr es ati hi i sgrifennu y delyneg ... Fe'i canwyd gannoedd o weithiau heb i neb sylweddoli mai mewn digofaint y cyfansoddwyd hi! ${ }^{17}$ 
A'r dyfyniad hwn wedi ei godi o raglen deledu a wnaed ar ddiwedd un ei oes, cyfnod pan ystyrid ef ei hun yn 'ŵr hen a pharchus iawn', mae'n ddadlennol mai ansoddair negyddol i ddilorni eraill o hyd yw 'parchus' gan Cynan ac nad oes unrhyw awgrym o hunanadnabyddiaeth yn y disgrifiad.

Gohebydd ifanc wyth mlynedd yn iau nag ef o'r enw J. T. Jones (1903-85) - John Tudor Jones neu John Eilian - oedd cyswllt Cynan yn y Western Mail, ac ar dudalennau'r papur cenedlaethol hwnnw y cyhoeddwyd o leiaf saith o'i gerddi rhwng Ionawr 1923 a Rhagfyr 1924. ${ }^{18}$ Yn eu plith mae rhai sydd wedi parhau ac sy'n tystio i'r melodedd canadwy a gysylltir gydag amryw o'i gerddi gan eu bod wedi hen ymsefydlu'n rhan o'r repertoire gwerin ('Y Ferch o Dy'n y Coed'), cerdd dant ('Defaid ac Ŵyn'), unawdol ('Tresaith'), a phop ('Aberdaron').

Gydag Edward Prosser Rhys (1901-45), gŵr ifanc arall a oedd chwe blynedd yn iau na Cynan, byddai John Eilian yn cyhoeddi Gwaed Ifanc cyn diwedd 1923. Dyma ddarn o lenyddiaeth ymosodol a âi ati i godi twrw; a'i theitl yn amlygu'r 'ifanc', mae angen ei deall yng nghyd-destun y gwrthdaro rhwng yr hen a'r ifanc a gododd yn sgil y Rhyfel Byd Cyntaf. Yn Gymraeg, W. J. Gruffydd yn '1914-1918: Yr Ieuainc wrth yr Hen’ sy’n rhoi’r mynegiant mwyaf diflewyn-ar-dafod i'r gwrthdaro hwn. Efallai'n ddealladwy a hithau wedi ei chyfansoddi ar ddydd y cadoediad yn Nhachwedd 1918, mae'r gerdd yn pegynu'n eithafol rhwng yr hen do llygredig - a fanteisiodd ar eu hawdurdod a'r ymddiriedaeth a roed ynddynt - a'r to iau a yrrwyd ganddynt 'Fel gyr o anifeiliaid mud' i frwydro yn y rhyfel. ${ }^{19}$ Yr 'hen ddynion blin' a dargedir ganddo yw'r '[P] enadur gwlad, cynghorwr plwy', | Arglwydd yr aur, a thorrwr glo' a'r cyfan yn ddiwahân yn cael eu cyfri ganddo'n fudrelwyr. ${ }^{20} \mathrm{O}$ gymharu, yr ifainc yw'r rhai gonest, delfrytgar a gadwodd eu hygrededd moesol, ac er eu bod wedi eu lladd, maent o leiaf yn gallu diolch nad ânt 'byth fel chwi, | Wrth gofio nad awn byth yn hen'. ${ }^{21}$ Yn ôl dehongliad Gruffydd ohono, mae henaint yn gyflwr tocsig a'r 'ifanc' yn nheitl cyfrol Prosser Rhys a John Eilian yn air llwythog.

Mewn sgwrs rhwng y ddau yn Eisteddfod yr Wyddgrug, cododd Prosser Rhys awydd mawr ar Cynan i ddarllen cerddi'r gyfrol fel yr addefodd mewn llythyr at John Eilian ar y pryd: 'Clywaf fod yna lawer o bridd y ddaear ynddi, - peth digon amheuthyn yng Nghymru heddiw ysywaeth. Ei hadolygu? Â phleser, a'i hystyried yn fraint cael fy nghysylltu mewn unrhyw 
fodd a chyfrol a dyrr gymaint o dir newydd. ${ }^{22}$ Ac yntau'n gynrychiolydd amlwg o'r ifainc a aberthodd yn y rhyfel, cyhoeddwyd adolygiad Cynan ar dudalennau'r Brython ar 8 Tachwedd lle'i hamddiffynnodd yn ddiamwys ar sail ei hannibyniaeth a'i gonestrwydd:

Dewisodd dau fardd ifanc feddwl drostynt eu hunain a mynegi eu meddwl yn eu ffordd eu hunain. Ac i lawer ohonom dyna bechod anfaddeuol mewn dyn ifanc.

Cofiaf am N.C.O. o'r hen deip yn taranu uwch fy mhen pan oeddwn yn y fyddin, 'You're not supposed to think. You're $[s i c]$ job is to obey orders.'

Gŵwyr pawb fu yn y fyddin mai dyna'r llwybr esmwythaf - cyflawni pob gorchymyn heb holi dim, a gwylio er popeth gadw i fyny'r defoda a'r traddodiadau.

Wrth gwrs dyna'r ffordd esmwythaf trwy fywyd hefyd.

Ond beth pan gyfyd dyn a wrthyd beidio â meddwl ac a wrthyd ddal ei dafod? Beth pan gyfyd dyn a wrthyd gymryd popeth yn ganiataol? Beth pan gyfyd dyn a wrthyd ganlyn y lliaws a phlygu i Baal?

Brensiach, dyna'r saim yn y tân wedyn!

Y funud y dechreua dyn feddwl drosto'i hun, y mae'n agored i’w gamfarnu.

Gonestrwydd y gyfrol yw ei grym

Nid yn ddiniwed y tynnodd Cynan ar ei brofiad milwrol yn y dyfyniad hwn i ddelweddu ei ddameg ond hefyd i ychwanegu cyfiawnhad moesol at ei safbwynt yn wyneb unrhyw ymosodiadau ar yr ifainc - beirdd Gwaed Ifanc ac yntau - o du'r hen feirniaid. Pa hygrededd erbyn 1924 fyddai i orchmynion 'N.C.O. o'r hen deip' heb sôn am barch i'w eiriau? Gwnaeth Cynan rywbeth tebyg sef dwyn ar ei brofiad fel un o oroeswyr y rhyfel yn 'Balâd' gyda'i hisdeitl ergydiol: 'Anerchiad nas clywir oddiar risiau Cofeb yr un o'n Colegau, Ddydd y CadOediad'. Yn honno, gweithreda fel '[c]ennad' ar ran y 'bechgyn' na ddaeth yn ôl o'r rhyfel ac ymuniaethu gyda 'Hen ffrindiau'r ffos' a gyferbynnir a '[ph]lant' y 'budrelwyr', ${ }^{23}$ ac fel 
Wilfred Owen o'i flaen yn 'Dulce Et Decorum Est', ${ }^{24}$ dyfarnu'n '[g]elwydd' y geiriau a naddwyd ar gerrig coffa sef " MELYS YW MARW DROS EIN GWLAD”".25

Fe gynhyrfodd Gwaed Ifanc y dyfroedd llenyddol a bron na fuasai'n siom i'w hawduron dewr pe na bai hynny wedi digwydd. Delweddaeth 'Y Canibal Anystyriol', er enghraifft, a dramgwyddai R. Meirion Owen o Glynnog, gŵr a gâi drafferth meddwl yn drosiadol: ar ôl dyfynnu ohoni - 'Can's euthum innau'n gannibal, | Bwyteais Gorff ac yfais Waed' dymunai gael gwybod 'ar ba sail y mae awdur y llinellau hyn yn cludo'r fath wenwyn i amgylchiad mor santaidd' â'r cymun. ${ }^{26}$ Wrth ymateb iddo yn y rhifyn dilynol, cydnabu John Eilian fod y gyfrol wedi ennyn cryn wahaniaeth barn ymhlith adolygwyr cyn nodi'n ddigon ddiedifar os hunanfodlon fod gan yr awduron "y boddhâd o wybod bod "gwaed ifanc" Cymru, o leiaf, yn cydymdeimlo â'n gwaed ifanc ninnau'. ${ }^{27}$ Unwaith eto, felly, lleolir yr ymateb i'r gyfrol yng nghyd-destun y tyndra rhwng dwy genhedlaeth a ddeilliodd o gyfnod y rhyfel. Hynny yw, a beirniadaeth yr hen yn amherthnasol, cael cefnogaeth y rhai ifainc moesol gywir oedd yn bwysig.

Ymuniaethodd Cynan ymhellach â'i gyd-feirdd ifainc drwy'u hamddiffyn mewn cerdd a gyflwynir, o leiaf yn fersiwn Caniadau Cynan ohoni, i John Eilian a Prosser Rhys sef 'Yr Hen Ysgol wrth y Newydd'. Onid yw ei theitl yn gyfeiriadol ac yn adleisio, ond yn gwyrdroi'r un pryd, 'Yr Ieuainc wrth yr Hen' Gruffydd? Yng ngherdd Cynan, rhoddir llais i'r to hŷn sy'n ceisio torri crib y rhai ifainc drwy honni'n nawddoglyd ac yn hunanfodlon y bydd '[b]echgyn gwyllt' heddiw yn gocyn hitio fel hwythau i feirdd newydd y dyfodol:

Y mae dydd i ddod pan fyddwch

Fel nyni mewn blin lonyddwch

Wedi oeri o'r ifanc waed,

Wedi blino o'r buain draed.

Ac yn nyddiau eich sarhaed

Chwithau hefyd a geryddwch. ${ }^{28}$

Yn y dyfodol, mynn y bydd eu beiddgarwch wedi hen ddyddio: 'i'r newydd do annheg, | Pethau dof a fydd eich rheg | A'ch sgrifennu "diawl" am "diafol". ${ }^{29}$ Ac mewn cerdd arall a berthyn i'r un cyfnod - cyhoeddwyd 'Rondo' ac iddi'r is-deitl 'Cyflwynedig i Ieuenctid Cymru sydd efallai yn gweled ymhellach na'u Ceryddwyr' ar dudalennau'r Western Mail ar 
21 Ionawr 1924 - mae'n ymuniaethu drachefn gyda'r ifainc amharchus. Fel y dychmygodd ei hun yn 'hen a pharchus' yn 'Aberdaron' bron flwyddyn ynghynt, felly hefyd y cyflwyna ddarlun cartŵn ohono'i hun 'yn hen bregethwr piwus, croes | Heb destun ond "chwareuon gwag yr oes"' yn y gerdd hon. ${ }^{30}$ Unwaith eto, cyflwr sy'n cyfleu syrthni a chonfensiynoldeb yw henaint, clefyd i warchod rhagddo.

Ond storm mewn cwpan oedd helynt Gwaed Ifanc o gymharu â'r sgandal genedlaethol pan enillodd Prosser Rhys y goron yn Eisteddfod Pont-y-pŵl yn Awst 1924 gyda phryddest a archwiliai'n eofn thema rhyw a disgrifio cariad hoyw. Ac unwaith eto roedd y drindod hon o feirdd ifainc - Cynan, Prosser Rhys a John Eilian - yn gefn i’w gilydd oherwydd nid yn unig y byddai'r tri yn rhannu llety yn yr un brifwyl, ond byddai Cynan yntau'n ennill y gadair a hynny gydag 'awdl' y bu dadlau fyth ers hynny ai awdl oedd hi o gwbl.

Dyma gyrraedd felly'r Clarence Hotel ym Mhont-y-pŵl a Cynan erbyn hynny wedi cael cadarnhad, nid drwy swyddfa'r Eisteddfod ond drwy John Eilian, mai ef fyddai'n hawlio'r gadair a Prosser Rhys y goron:

Melys fydd olrhain y pethau sydd y tu hwnt i'r llen pan gyrhaeddom Bont y Pŵl. Dywedwch eich bod i aros gyda Phrosser yn y Clarence Hotel. Ardderchog! Oherwydd gofynais iddo bookio drosof finnau hefyd, ac felly bydd yno driawd go hynod! Edrychaf ymlaen yn eiddgar at eich cyfarfod, a chael llawer o'ch cwmni yr wythnos nesaf yma. ${ }^{31}$

Drannoeth yr Eisteddfod, anfonodd eto at John Eilian i ddiolch iddo am ei drefniadau:

Nid trefniadau yn unig bid sicr, ond y peth byw hwnnw hefyd a gafodd Emrys yng nghyfeillach y saint:

'O mor hoff yw cwmni'r brodyr

Sydd a'u hwyneb tua'r wlad,

Heb un tafod yn gwenieithio

Heb un fron yn meithrin brad. ${ }^{32}$

Gwyn ei fyd y pryf ar y wal yn ystod seiadau'r Clarence Hotel wrth i'r tri chyfaill drafod eu cyfansoddiadau yn ddilyffethair a rhoi'r byd yn ei le. Pa gynllunio a chynllwynio a aeth 
ymlaen? Ac onid yma y câi Cynan ddiosg yn llwyr ei goler wen a rhoi rhwydd hynt i'w anian greadigol, cael byw fel bardd yng nghwmni dau enaid hoff cytûn? 'Haerasom fod y byd yn ddrwg i'w fôn; | Mynasem gael y byd o'i fôn yn dda' meddai Prosser Rhys mewn rhan o'i bryddest ddadleuol, ${ }^{33}$ ac ymdeimlir yn yr ohebiaeth rhwng Cynan a John Eilian o'r cyfnod hwn ag antur a chyffro'r tri delfrydwr ifanc. Kate Crockett sy'n sôn yn ei chyflwyniad ardderchog i fywyd a gwaith Dylan Thomas ei fod wedi marw 'fel seren roc, ar ôl cael ei daro'n wael yn y Chelsea Hotel yn Efrog Newydd, man eiconig yn hanes diwylliant poblogaidd, a'i gysylltiadau ymhen blynyddoedd wedyn â Bob Dylan, Leonard Cohen, Patti Smith a Sid Vicious'. ${ }^{34}$ O gymharu â deiliaid diweddarach y Chelsea Hotel, ni falwyd yr un gitâr fas na thaflu'r un teledu drwy ffenest gan yr un o breswylwyr y Clarence Hotel yn Awst 1924, ond anodd dychymgu cwmni mwy herfeiddiol a delwddrylliol o rocfeirdd wedi ymgasglu dan yr unto ym Mhont-y-pŵl ar y pryd.

Beth felly am 'awdl' 1924? Gyda thestun mor anaddawol ag 'I'r Duw nid Adweinir', efallai nad yw'n syndod mai cystadleuaeth symol a gaed am y gadair a deuddeg yn unig wedi cynnig amdani, dau ohonynt yn Saesneg. Bu Cynan hefyd yn ffodus yn ei feirniaid, yn enwedig ei hen athro Cymraeg ym Mangor gynt, Syr John Morris-Jones, y pennaf awdurdod ar gerdd dafod a ddywedodd yn ei feirniadaeth ar gerdd 'Alastor':

Dyma awdl ar y testun. Ond nid pawb a gydnabyddai fod hon yn awdl o gwbl, canys cân yw hi ar fesur tri thrawiad, heb fwy o gynghanedd ynddi nag sydd yng nghanu Huw Morus, na chymaint ag sydd ym mugeilgerddi Edward Richard. Ond y ddeddf sydd mewn grym yw'r penderfyniad a basiwyd yn Eisteddfod 1819, fod rhyddid i'r beirdd o hynny allan, yng nghystadleuaeth y gadair, ganu ar y mesur a fynnent o 'ddosbarth Morgannwg,' sef y dosbarth a ddyfeisiodd Iolo'i hun, ac y llwyddodd i berswadio beirdd yr oes honno mai'r 'hen ddosbarth' oedd. ${ }^{35}$

Ac yntau'n un o bennaf cynrychiolwyr ysgolheigion y brifysgol a oedd â'u llach ar y pryd ar ffug hynafiaeth yr Orsedd a ddyfeisiwyd gan Iolo Morganwg, mae'n eironig gweld MorrisJones yn cyfeirio at Iolo o bawb i roi carn i'w ddadl a chyfiawnhau cadeirio Cynan. Ond Morris-Jones lai deddfol a glywir, ac er nad oes awgrym ei fod yn ildio i heresi Islwyn drwy roi i'r awen 'dragwyddol heol', nid yw chwaith fel petai'n mynnu mai ar sail ei gallu i gydymffurfio â rheolau yr oedd barnu gwerth barddoniaeth o reidrwydd: 
Ond fel darn o farddoniaeth y mae'r gân hon yn rhagori tu hwnt i fesur ar ddim sydd yn y gystadleuaeth. Y mae ei hiaith yn dda . . . Y mae swyn rhyfeddol yn y gerdd; y mae hi'n ddarlun byw, ac yn gyfanwaith cwbl ynddi ei hun . . . y mae'r awdl yn union ar y testun; y mae hi'n darlunio bywyd, ac nid yn traethodi; fel barddoniaeth y mae hi'n rhagori'n fawr ar y lleill, ac y mae ei chynganeddiad yn gyfreithlon yn ol llythyren y ddeddf. Gan hynny, cadeirier Alastor. ${ }^{36}$

Broliodd un o'r beirniaid eraill, Elfed, 'newydd-deb a swyn y gerdd' ac 'fel llenyddiaeth gain' roedd hi 'ddigon o flaen yr un cystadleuydd arall'. ${ }^{37}$ Cytunai'r trydydd beirniad yntau, T. Gwynn Jones, nad oedd 'amheuaeth yn y byd nad dyma'r darn goreu o farddoniaeth yn y gystadleuaeth ar y testun' a bod y gerdd yn 'ddarn o lenyddiaeth a ddarllenir ac a ddyellir, ac a rydd wir bleser i ddyn'. ${ }^{38}$

Mewn adroddiad sy'n canmol a cheryddu am yn ail, fel hyn yr adroddwyd am lwyddiannau Eisteddfod Pont-y-pŵl ar dudalen flaen Y Brython, 14 Awst 1924:

Achoswyd cryn synnu a siarad ynglŷn ag awdl y gadair a phryddest y goron eleni - ffurf y naill a chynnwys y llall. Nid pawb wyddai fod Cynan yn ymhel a'r cynghaneddion o gwbl . .

Dewisodd Cynan ei fesur yn bwyllog, a chan farnu mai hwn oedd y mwyaf manteisiol i'w bwrpas ef. Drwy wneuthur hynny dangosodd annibynaeth na fynnai blygu i gyfansoddi yn y ffordd debycaf o ennill ar ei feirniaid, eithr i'r gwrthwyneb . . .

Ond nid felly y gwelai pawb bethau, gan gynnwys Eifion Wyn, bardd y ffolodd Cynan yn ifanc ar ei delynegion ac un a fu'n ei roi ar ben y ffordd gyda'r cynganeddion. Fel hyn y cwynodd y mentor am ei ddisgybl mewn llythyr at gyfaill: 'Cafodd Cynan y Gadair am gerdd sydd yn fwy o bryddest nag o awdl. Cyfleus iawn oedd syrthio'n ol ar fesur y “tri thrawiad” i guddio'i ddiffyg medr fel cynghaneddwr. ${ }^{39}$ Ac mor ddiweddar â 1958 roedd Iorwerth Peate yn dal i edliw ei gadair i Cynan: un o fesurau'r canu rhydd oedd y tri thrawiad 'a'r gynghanedd yn addurn ychwanegol yn unig' ${ }^{40}$ meddai mewn gohebiaeth rhyngddo a David Thomas, gan ychwanegu nad oedd gan Cynan 'hawl i ddisgwyl yr edrychid ar ei gerdd fel awdl'. ${ }^{41}$ Flwyddyn yn ddiweddarach ac yntau'n rhoi trefn ar Cerddi Cynan, yn yr adran 'Pryddestau' y cynhwysodd 'I'r Duw Nid Adweinir', arwydd prin os cynnil, efallai, i Cynan gydnabod cywirdeb ei feirniaid. 
Yn ogystal â Meuryn, un o bennaf colbwyr beirdd ifainc y cyfnod oedd gŵr o'r enw y Parch. W. A. Lewis, sylwebydd crintach a bychanus: 'Am y Cadeirio,' meddai, 'yr oedd yn fflat. Y dyddiau gynt, byddai'r dorf fawr yn ferw drwyddi gan gywreinrwydd a chwilfrydedd ... Oni wyddai pawb ers dyddiau mai Cynan oedd i'w gadeirio?' ${ }^{42}$ Gor-ddweud, efallai, ond a Cynan wedi cael clywed o flaen llaw gan y newyddiadurwr John Eilian mai ef a enillai, gellid rhoi rhyw goel ar yr awgrym. Ond wrth droi ei sylw at seremoni'r coroni yr aeth W. A. Lewis i hwyl wirioneddol:

Galwyd ar ddau o'n prifeirdd i gyrchu'r 'gwron' i'r llwyfan adeg y coroni, sef Pedrog a Chynan. Nid oedd Cynan ar y llwyfan nac mewn urddwisg (er ei fod mewn urddwisg y bore hwnnw) ond dacw fo - yn ei 'ddillad ac yn ei iawn bwyll' gellir tybio - yn cadw cwmni i'w gyfaill Prosser Rhys, a bu'n rhaid i Pedrog fynd ei hun, a dyna olwg ddi-urddas oedd ar y tri yn ymlwybro i'r llwyfan - bardd yn un fraich i'r buddugwr, a dyn cyffredin yn y fraich arall! Wedi cyrraedd hyd at yr Archdderwydd cymerodd Cynan ei le ymysg ei frodyr gorseddol heb wisg y briodas, er mai ef drachefn, gyd â Chrwys, oedd i annerch y bardd coronog. I mi y mae holl ymddygiad nifer o'n beirdd a'n llenorion ieuainc at ddeddf ac arferiad Gorsedd a Steddfod, at lên a barddas, at gyfundrefnau cymdeithas a chrefydd, yn sawru'n gryf o Anarchiaeth. ${ }^{43}$

Ond nid yn unig yr anfarwolwyd Cynan a Prosser Rhys ag amharchusrwydd drwy eu cyfri'n anarchwyr, trodd beirniadaeth W. A. Lewis yn fwy personol byth pan gyfeiriodd at 'freaks of nature, ac os ydyw Prosser Rhys yn un o'r freaks hynny, y mae'n wrthrych tosturi'. ${ }^{4}$ Arweiniodd hynny at ymddiheuriad ar dudalennau'r Brython, ac awgrymir bod Prosser Rhys 'wedi ennill achos llys yn erbyn y gŵr hwn am athrod'. ${ }^{45}$ Yn ei amddiffyniad ef o Prosser Rhys yn wyneb ymosodiad ciaidd W. A. Lewis, hawliodd bardd ifanc eofn arall o'r enw Caradog Prichard na allai 'Mr Lewis gredu bod y gerdd yn bortread cywir o brofiad dyn' a hynny am na 'welodd ac ni phrofodd fywyd yn null bardd'. ${ }^{46}$ Fel Shelley a ddyfarnodd feirdd yn ddeddfwneuthurwyr anghydnabyddedig y byd, gosododd yntau gyfraniad a gwelediad beirdd ar wastad uwch na'r cyffredin, hyd yn oed uwchlaw'r 'Parchedig' o flaen enw W. A. Lewis. Yng ngoleuni'r croestyniadau ynddo rhwng y bardd a'r pregethwr, oni roddodd awgrym felly ddeunydd i Cynan gnoi cil arno? Ac yntau ar fin ymsefydlu'n un o leisiau barddol diffiniol ail hanner y 1920au drwy ennill y goron dair gwaith yn olynol, does dim 
syndod deall bod triawd y Clarence Hotel wedi tyfu'n bedwarawd yn y man a bod Caradog Prichard wedi ei dderbyn yn gyflawn aelod o'r cwmni eisteddfodol dethol a gyd-letyai.

Nac anghofiwn chwaith mor fentrus, yn wir, mor amharchus oedd cynnwys llawer o awdl ddeialogaidd Cynan. Ymryson a gaed yn y fersiwn eisteddfodol a'r Parch. A. E. Jones wedi gweithredu'n ddiogel drwy godi ei destun o ail lyfr yr Actau, pennod 17 lle y ceir gohebiaeth rhwng Glaucon yr Epicuriad a'i gyfaill Bion y Stoic. Ar sail y llythyrau sy'n mynegi eu safbwyntiau cyferbyniol y codwyd y gerdd. Llais rheswm a deall a glywir gan Bion sydd hefyd yn ychwanegu dimensiwn ysbrydol at y gerdd drwy gyfeirio at ymweliad Paul ag Athen i bregethu am Grist. Ond pan aeth Cynan ati i baratoi Caniadau Cynan ar gyfer ei chyhoeddi yn 1927, un ochr y ddadl a gyflwynwyd ganddo sef detholiad o adran gyntaf y gerdd a llais Glaucon yn unig, er enghraifft 'Pa les wna'ch colegau a'u holl ddamcaniaethau | A'ch cyrddau i ddadleuon crefyddau?', ${ }^{47}$ geiriau rhyfedd o enau gŵr gradd a gweinidog at hynny. Felly hefyd y canu hwn, nid i'r ysbryd ond i'r cnawd:

Ond tra byddo'i gwefus mor goch ac mor felys A'r mefus, a hanner esgeulus ei gwallt, A minnau'n ei blethu a'm gwaed yn cyflymu Pan ddringom i garu mewn gorallt ${ }^{48}$

Efallai na wneir mwy na boddio'r synhwyrau fel y gwnaeth John Morris-Jones yn 'Penillion Omar Khayyâm' yn 1907 a dathlu tegwch y funud fel R. Williams Parry yn awdl 'Yr Haf' yn 1910: ‘Ar fyrr dygir minnau ar alwad Yr Angau | I wylfro'r Cysgodau i liwiau y wlad. | Ond heddyw mae pleser, a serch, a phob mwynder. | Yf win oni alwer yr alwad. ${ }^{49}$ Ond er eu parchusrwydd, addysgwyr ac academwyr oedd y ddau hynny: ac yntau'n weinidog yr Efengyl, roedd Cynan yn hwylio'n o agos at y gwynt. Oedd, roedd ganddo ystyriaethau esthetig dros hepgor llais Bion o ddetholiad 1927 o'i gerdd: 'nid mor hawdd rhoi athroniaeth strict a di-liw y Stoic ar gân â rhoi athroniaeth yr Epicuriad,' meddai mewn llythyr at John Eilian ar y pryd.$^{50}$ Wedi dweud hynny, Caniadau Cynan oedd enw'r gyfrol, nid Caniadau'r Parch. A. E. Jones, ac onid oedd hefyd drwy'r detholiad yn datgan mai gydag ysbryd hedonistaidd Glaucon y cydymdeimlai'r bardd ifanc? 
Arwydd arall o Cynan y bardd yn arloesi ac yn torri ei gŵys ei hun yn y cyfnod hwn, yn mynegi ei annibyniaeth ac yn coleddu safbwyntiau, rhai amhoblogaidd os nad amharchus o reidrwydd, oedd ei gerddi cenedlaetholaidd. Ar 14 Mawrth 1924 yn y Western Mail y gwelodd 'Cwm Rhondda' olau dydd gyntaf, telyneg seml ond un sydd, dros flwyddyn cyn ffurfio'r Blaid Genedlaethol, yn rhoi mynegiant cynnar i'r math o bolisi cenedlaetholaidd dadleuol a fynegwyd yn Deg Pwynt Polisi 1933: 'Er mwyn iechyd moesol Cymru ac er lles moesol a chorfforol ei phoblogaeth, rhaid yw dad-ddiwydiannu Deheudir Cymru. ${ }^{51}$ Drwy ddelweddaeth feiddgar gresynir at y modd y difethwyd yr ardal gan ddiwydiant gan bwyntio bys cyhuddgar at y rhai 'a dreisiodd lendid | Morwynig wyllt y Cwm, | A'i gadael yn anafus, | Yn aflan ac yn llwm'. ${ }^{52}$ Cyn i'r beirniad yn Eisteddfod Treorci 1928 omedd i Gwenallt y Gadair oherwydd beiddgarwch awdl 'Y Sant' a chyn iddo gyhoeddi gyntaf yn 1936 ei soned 'Cymru' a'i portreadai fel '[p]utain fudr y stryd â'r taeog lais', ${ }^{53}$ arloesodd Cynan yn y soned “"Â Chas Perffaith"' nad oes dim yn gysurus am ei delweddaeth na'n gymodlon am ei hagwedd:

Cashaf di, Gymru, am dy butain wên

I'r estron taeog rhwng dy fronnau di.

Cashaf di am it lygru d'enw hen

Yn wasaidd erddo, a'th frenhinol fri.

Cashaf di, am it wadu breiniol iaith ${ }^{54}$

Nid syndod i J. Gwyn Griffiths, mewn adolygiad ar Cerddi Cynan yn 1960, gyfeirio at y soned hon fel un 'epilgar ei dylanwad'. ${ }^{55}$ Trewir nodyn ymgyrchol yn 'Balâd Dyffryn Ceiriog' sy'n cyfeirio at fwriad Corfforaeth Warrington yn 1923 i foddi dros 13,000 o erwau Dyffryn Ceiriog a chodi argae er mwyn sicrhau cyflenwad dŵr i'r dref honno; byddai pentrefi Llanarmon a Thregeiriog wedi diflannu dan y don. Mewn ymgyrch sy'n rhagfynegi Tryweryn yn y 1950au a'r 1960au, gwrthwynebwyd y mesur gan holl Aelodau Seneddol Cymru a Lloyd George yn amlwg yn eu plith, ond y tro hwn, llwyddwyd i drechu'r mesur. ${ }^{56}$

Beth waeth fod dychryn dan fy ais?

Beth waeth fod deigryn ar fy ngrudd Wrth synfyfyrio ar y trais

A llwytho 'nodrefn? Daeth y dydd

I ffoi o flaen y llid a fydd. 
Pwy ydym ni i gadw stŵr?

Ffarmwr neu ddau, a gôf, a chrydd.

Cleddwch ein cartref dan y dŵr..$^{57}$

A daw i ben gyda'r siars hon: 'Gymro, os ydwyt eto'n rhydd, | Ymorol. Neu, fe gladd y gŵr, | Nad yw Clawdd Offa iddo'n lludd, | Holl Gymru Fu o dan y dŵr. ${ }^{58}$ Diddorol nodi, er bod hynt Tryweryn yn fater ysol o gyfredol adeg ei chyhoeddi, mai'n ofer y chwilir Cerddi Cynan yn 1959 am gerdd gyfatebol i'r un am Ddyffryn Ceiriog.

Un agwedd arall ar Caniadau Cynan sy'n awgrymu'r pwys a roddodd ar ei hunaniaeth fel bardd oedd ei gyfieithiadau o waith beirdd eraill, yn eu plith rai cyfoes a ffasiynol fel Aldous Huxley, Thomas Hardy, Rabindranath Tagore, Walter de la Mare, A. E. Housman a Katherine Tynan. Diau bod hyn wedi ychwanegu rhyw wedd soffistigedig at y gyfrol, ac yn achos yr Asiad Tagore - yr awdur cyntaf y tu allan i Ewrop i ennill Gwobr Nobel am Lenyddiaeth - awgrym o ryngwladoldeb eangfrydig. At hynny, awgrymant gwmni o eneidiau hoff, cytûn y gallai ymuniaethu â nhw, cwmni dethol a gadarnhai ei neilltuolrwydd yntau fel bardd.

A Cynan erbyn hynny yn ei dymor cyntaf fel Archdderwydd, ceisiodd grisialu yn 1951 bwysigrwydd yr Eisteddfod iddo ar ôl ei dilyn yn ddi-fwlch ers deng mlynedd ar hugain:

... mi addefwn i chwi'n rhwydd fod 'cwmni'r pererinion wrth fy modd'.

Ie siwr, yr ochr gymdeithasol i'r Eisteddfod - yr 'wythnos yn yr haf' gyda'r drws dihangfa i ambell 'artist yn Philistia'. Am rai blynyddoedd bu tri bardd ifanc yn cydletya'n gyson dros bob Eisteddfod Genedlaethol - Prosser Rhys, John Eilian, a minnau, ac yn ddiweddarach, Caradog Pritchard $[$ sic $]$.

Coffa da am y nosweithiau o drafod a dadlau llenyddiaeth gyda'n gilydd . . . coffa da hefyd am ein nosweithiau llawen, yng ngwir ystyr y gair. Nid ryw [sic] gyngherddau sâl i godi arian at achosion da, ond cynulliadau preifat drwy drefniant pedwar bardd ifanc, - a thalentau gorau'r Eisteddfod wedi dod ynghyd ar wahoddiad i'w hadlonni ei gilydd fel cwmni diddan. ${ }^{59}$ 
A dyna ni'n ôl gyda chwmni'r Clarence Hotel ac arwyddocâd y gwmniaeth honno i Cynan.

Cyfnod o ryw bymtheg mlynedd, rhwng 1916 a 1931, yw cyfnod mwyaf cynhyrchiol Cynan fel bardd; bardd achlysurol a fu ar ôl hynny a chasglodd ei gerddi ynghyd ddiwedd y 1950au yn Cerddi Cynan. Ond cyn hynny, rhoes un mynegiant cofiadwy i'r bardd anniddig a thramgwyddus o'i mewn yn Eisteddfod Bangor 1931 lle'r enillodd gyda'r ddrama Hywel Harris ond hefyd am gyfieithiad o 'Cân y Caniadau'. Wrth ddiolch iddo yn 1940 am gael benthyg copi o'r gerdd honno, teimlai I. D. Hooson ei fod 'yn or-feiddgar mewn rhannau o'r gân', ${ }^{60}$ ac at y ffaith fod 'rhai o'i ddelweddau yn rymus rywiol' ${ }^{61}$ y cyfeiriodd Harri Gwynn yntau wrth adolygu Cerddi Cynan yn 1960. Am 'Y Dyrfa', y bryddest a enillodd i Cynan ei drydedd goron, unwaith eto, daeth helynt i'w ganlyn pan wrthododd yr emynydd-bregethwr Moelwyn fynd i ben y llwyfan gyda'i gyd-feirniaid am na chredai fod defnyddio barddoniaeth i drafod rygbi yn briodol heb sôn am fod yn barchus. Ond gellid dadlau, o ran ei heffaith bellgyrhaeddol, mai dyma gerdd bwysicaf Cynan yn yr ystyr ei bod hi wedi lledu tiriogaeth destunol barddoniaeth Gymraeg a pharatoi'r ffordd ar gyfer cerddi seciwlar a roddodd lais Cymraeg i'r diwylliant Eingl-Americanaidd fel y rhai am Marilyn Monroe, John F. Kennedy ac Elvis Presley gan Gwyn Thomas yn y 1960au a'r 1970au.

Yn 1931 byddai Cynan yn ymadael â'r weinidogaeth gyfundrefnol, er na fyddai'n rhoi'r gorau i bregethu chwaith: byddai'n anarferol iddo gael Sul gwag weddill ei oes. Efallai fod y rhwyg honno'n anorfod ac yn arwydd o'i fethiant i gysoni rhwng y gweinidog a'r bardd. Ar ei swydd fel bardd y rhoddai'r bri mwyaf ac felly y carai gael ei gofio a barnu yn ôl tystiolaeth brawddeg gyntaf ei hunangofiant sy'n cyfeirio ato'i hun fel '[b]ardd o Gymro'. ${ }^{62}$ Yn sgil ei benodiad i'r brifysgol ym Mangor, cafodd ryddid i dorri ei gŵys ei hun a rheoli ei dynged ei hun i raddau helaeth iawn, i roi mynegiant llawn i'r ysbryd bardd o'i mewn, fel petai. Ac ar bapur o leiaf, rhyddhâi'r swydd newydd amser iddo, ffactor ymarferol yr oedd Morgan Humphreys wedi cyfeirio ato mewn adolygiad nodweddiadol graff o Caniadau Cynan yn 1927:

Gwr prysur yw Cynan ... y mae y rhan fwyaf o feirdd Cymru yn rhywbeth, ac weithiau yn llawer o bethau, heblaw beirdd. Hwyrach y byddai'n ormod dywedyd na all dyn sydd yn llawer o bethau heblaw bardd fod yn fardd mawr, ond y mae'n weddol sicr fod rhyw raddau o 
ymneilltuo, rhyw gymaint o lonyddwch, rhyw gymaint o unigrwydd yn angenrheidiol. Teimlaf mai o'r unigrwydd hwnnw y seinia llais Cynan gliriaf. ${ }^{63}$

Ac eto, yn yr ystyr mai ffrwyth y croestynnu a brofai rhwng ei swyddogaeth gyhoeddus fel gweinidog a'i ddyheadau mewnol fel bardd oedd amryw o'r cerddi a ysgrifennodd yn ystod y 1920au, efallai fod rhoi'r gorau i'w weinidogaeth wedi tawelu'r tyndra a borthai ei awen ac mai dyna un o'r ffactorau a arweiniodd at ei dawelwch cymharol fel bardd ar ôl dechrau'r 1930au. At hynny, roedd yn artist a fwynhâi heriau uchelgeisiol a chyda cyfnod ei gyflawniadau ym mhrif gystadlaethau'r Eisteddfod ar ben, yn awyddus i sianelu ei egni creadigol i brosiectau eraill. Dichon fod y pasiantau mawreddog y bu ynglŷn â nhw yng nghastell Conwy a Chaernarfon yn ail hanner y 1920au, rhai a gynhwysai gast o gannoedd ac a ddenai gynulleidfaoedd o filoedd, wedi ei ddeffro i botensial gweledol a dramatig yr Orsedd. Profodd boblogrwydd mawr fel bardd, ond fel ffigwr cyhoeddus, nid oedd arno ofn amhoblogrwydd chwaith: oherwydd ei hunanhyder, roedd yn argyhoeddedig mai ef oedd yn iawn. Felly mae'r annibyniaeth barn a ddangosodd drwy'i benderfyniad dadleuol i sicrhau rhan ganolog i'r Orsedd yn Arwisgiad 1969 yn gyson ag ysbryd eofn rhai o gerddi'r 1920au.

Rhag ofn i ddau rocfardd arall y Clarence Hotel fynd yn angof, bu farw Prosser Rhys yn gynamserol yn 1945 ac o golofnau papurau'r Herald, cynhaliodd John Eilian, y Tori sefydliadol erbyn hynny, ymgyrch ddidostur yn erbyn pennaf cynrychiolydd protestwyr y 1960au, Dafydd Iwan. Ar dudalennau Tafod y Ddraig a Lol yn ail hanner y 1960au, ffigwr cartŵn oedd Cynan, ond o ran ei argyhoeddiad brenhinol a'i frwdfrydedd tryloyw drosti, o leiaf arhosodd yn driw iddo'i hun. Efallai mai dyna sy'n egluro, er ei fod erbyn diwedd ei oes wedi troi'n feirniad llym o'r to ifanc protestgar, pam na allai weld ynddo'i hun unrhyw ymgorfforiad o'r union fath o gymeriad adweithiol y bu yntau'n ei dro yn ei watwar a'i gartwneiddio yn rhai o gerddi'r 1920au.

Bangor

GERWYN WILIAMS

\footnotetext{
* Fersiwn estynedig o Ddarlith Goffa Syr Thomas Parry-Williams a drefnwyd gan y
} Ganolfan Uwchefrydiau Cymreig a Cheltaidd a'i thraddodi yn Llyfrgell Genedlaethol Cymru, 
25 Ebrill 2018. Diolch i’r Athro Dafydd Johnston, Cyfarwyddwr y Ganolfan, am y gwahoddiad.

${ }^{1}$ E. Morgan Humphreys at T. Gwynn Jones, 6 Hydref 1918, Casgliad T. Gwynn Jones, 2236, LlGC.

${ }^{2}$ Y Cyhoeddwyr, 'Rhagair', Telyn y Nos (Wrecsam: Hughes a'i Fab, 1921), t. iii.

3 'Nodion Llywarch Hen', North Wales Weekly News, 18 Awst 1921.

4 'Cynan', yn Gerwyn Wiliams (gol.), Gorau Cyfarwydd: Detholiad o Ddarlithoedd ac Ysgrifau Beirniadol Bedwyr Lewis Jones (Cyhoeddiadau Barddas, 2002), t.251.

${ }^{5}$ Gw. y ffotograff ‘Gyda'r Tywysog Charles yn Eisteddfod Y Fflint 1969', rhif 102, yn Ifor Rees (gol.), Bro a Bywyd Syr Cynan Evans-Jones 1895-1970 (Caerdydd: Cyngor Celfyddydau Cymru, 1982), t. 55.

${ }^{6}$ John Maxwell Jones, Cyfarwyddiadur Awduron Cymraeg Cyfoes (Philadelphia: cyhoeddwyd gan y golygydd, 1970), t. 13.

${ }^{7}$ Stephen King, The Brotherhood: The Secret World of the Freemasons (London: Panther Books, 1985), t. 3.

8 ‘Aberdaron', Caniadau Cynan (Llundain: Foyle’s Welsh Depot, 1927), t. 37.

9 'Y Nasaread', Caniadau Cynan, t. 33.

10 'Mab y Bwthyn', Telyn y Nos, t. 20.

${ }^{11}$ Cynan at R. Silyn Roberts, 12 Mawrth 1921, Papurau Silyn, 19590, Archifdy Prifysgol Bangor.

12 'Monastîr', Caniadau Cynan, t. 46.

13 'Y Tannau Coll', Caniadau Cynan, t. 97.

${ }^{14}$ T. H. Parry-Williams, Cerddi (Aberystwyth: Gwasg Aberystwyth, 1931), tt. 18-20 a tt. 214.

${ }^{15}$ Cynan at John Eilian, 22 Medi 1923, gohebiaeth ym meddiant Goronwy Tudor Jones.

${ }^{16}$ Gw. ei gerdd goffa iddo, 'The Roadmaker', Cerddi Cynan (Lerpwl: Gwasg y Brython, 1959), tt. 110-11.

${ }^{17}$ Cynan, Y Llanc o Lŷn, rhaglen deledu a ddarlledwyd ar BBC Cymru yn 1970. Ceir copi fideo o'r ffilm yn Archif Sgrin a Sain LlGC; fe'i hailddarlledwyd ar 15 Ionawr 1985 yn nghyfres Cyfle Arall S4C.

${ }^{18}$ Ymddangosodd 'Aberdaron' yn y Western Mail ar 17 Ionawr 1923; 'Rondo' ar 21 Ionawr 1924; 'Tresaith' ar 7 Chwefror 1924; 'Cwm Rhondda' ar 14 Mawrth 1924; 'Dinistr 
Senacherib' (Byron) ar 2 Mehefin 1924; 'Defaid ac Wyn' (Katherine Tynan) ar 5 Tachwedd 1924; ac 'Y Ferch o Dy'n y Coed' ar 20 Rhagfyr 1924.

${ }^{19}$ W. J. Gruffydd, '1914-1918: Yr Ieuainc wrth yr Hen', Ynys yr Hud a Chaneuon Eraill (Wrecsam: Hughes a'i Fab, 1963), t. 44.

${ }^{20}$ Ibid, tt. $44-5$.

${ }^{21}$ Ibid, t. 45.

${ }^{22}$ Cynan at John Eilian, 22 Medi 1923, gohebiaeth ym meddiant Goronwy Tudor Jones.

23 'Balâd', Caniadau Cynan, tt. 66-7.

${ }^{24}$ C. D. Lewis (gol.), The Collected Poems of Wilfred Owen (London: Chatto \& Windus, 1983), tt. 54-5.

25 'Balâd', Caniadau Cynan, t. 67.

${ }^{26}$ Y Brython, 6 Rhagfyr 1923.

${ }^{27}$ Y Brython,13 Rhagfyr 1923.

28 'Yr Hen Ysgol wrth y Newydd', Caniadau Cynan, t. 42.

${ }^{29}$ Ibid.

${ }^{30}$ Ibid, t. 63.

${ }^{31}$ Cynan at John Eilian, diddyddiad, gohebiaeth ym meddiant Goronwy Tudor Jones.

${ }^{32}$ Cynan at John Eilian, diddyddiad, gohebiaeth ym meddiant Goronwy Tudor Jones.

${ }^{33}$ E. Prosser Rhys, 'Atgof', yn J. M. Edwards (gol.), Cerddi Prosser Rhys (Dinbych: Gwasg Gee, 1950), t. 34.

${ }^{34}$ Kate Crockett, Mwy Na Bardd: Bywyd a Gwaith Dylan Thomas (Cyhoeddiadau Barddas, 2014), t. 154.

35 ‘Awdl y Gadair: “I'r Duw nid Adweinir"', yn E. Vincent Evans (gol.), Cofnodion a Chyfansoddiadau Eisteddfod Genedlaethol 1924 (Pontypwil), t. 7.

${ }^{36}$ Ibid, tt. 8-9.

${ }^{37}$ Ibid, t. 12.

${ }^{38}$ Ibid, tt. 16-17.

${ }^{39}$ Dyfynnwyd yn Peredur Wyn Williams, Eifion Wyn (Llandysul: Gwasg Gomer, 1980), t. 178.

40 Iorwerth Peate at David Thomas, 10 Hydref 1958, Papurau David Thomas, 19210, Archifdy Prifysgol Bangor.

${ }^{41}$ Iorwerth Peate at David Thomas, 14 Hydref 1958, Papurau David Thomas, 19210, Archifdy Prifysgol Bangor. 
42 Y Brython, 4 Medi 1924.

${ }^{43}$ Ibid.

${ }^{44}$ Ibid.

${ }^{45}$ Alan Llwyd, Blynyddoedd y Locustiaid: Hanes Eisteddfod Genedlaethol Cymru 1919-1936 (Cyhoeddiadau Barddas, 2007), t. 168.

${ }^{46} Y$ Brython,11 Medi 1924.

${ }^{47}$ Caniadau Cynan, t. 91.

${ }^{48}$ Ibid.

${ }^{49}$ Ibid, t. 93.

${ }^{50}$ Cynan at John Eilian, diddyddiad, gohebiaeth ym meddiant Goronwy Tudor Jones.

${ }^{51}$ Saunders Lewis, Canlyn Arthur (Aberystwyth: Gwasg Aberystwyth, 1938), t. 12.

52 'Cwm Rhondda', Caniadau Cynan, t. 32.

53 'Cymru', yn Christine James (gol.), Cerddi Gwenallt: Y Casgliad Cyflawn (Llandysul: Gwasg Gomer, 2001), t. 98.

${ }^{54}$ Caniadau Cynan, t. 27.

55 J. Gwyn Griffiths, 'Cerddi Cynan', Baner ac Amserau Cymru, 19 Mai 1960.

56 Tŷ’r Cyffredin. Hansard. [Ar-lein]. Ar gael:

http://hansard.millbanksystems.com/commons/1923/mar/13/private-business-

1\#column_1405, cyrchwyd 4 Medi 2018.

57 'Balâd Dyffryn Ceiriog', Caniadau Cynan, t. 69.

58 Ibid.

${ }^{59}$ Cynan, 'O Berthynas i’r Eisteddfod', Llafar, 1951, 135-6.

${ }^{60}$ I. D. Hooson at Cynan, 23 Gorffennaf 1940, Papurau Cynan, 22613, Archifdy Prifysgol Bangor.

${ }^{61}$ Harri Gwynn, 'Trwy’r Glust i'r Galon y Traidd Canu Cynan', Y Cymro, 24 Mawrth 1960.

62 'Hunangofiant Cynan: 1 Mabinogi', yn Ifor Rees (gol.), D̂̂w o Ffynnon Felin Bach (Dinbych: Gwasg Gee, 1995), t. 13.

63 'Wrth Fynd Heibio', 18 Gorffennaf 1927, toriad papur newydd ymhlith Papurau Cynan, 22575, Archifdy Prifysgol Bangor. 